\title{
A 'snowflake' geometrical representation for optimised degree six 3-modified chordal ring networks
}

\begin{abstract}
The performance parameters and properties of chordal rings have been researched extensively as models for parallel and distributed interconnection topology models since their founding in 1981. A chordal ring is modelled after a circulant graph, where its vertices represent processor nodes and its edges represent the links between them. Hence, its performance and properties of connectivity can be studied through graph theory. This research was aimed at the investigation of a new degree six chordal ring, the optimised degree six 3-modified chordal ring CHR6o3. A tree visualisation was constructed based on its connectivity to enable the generation of formulae for optimal diameter and average optimal path lengths. As the numbers of nodes further increased with its layers, the visualisation was found to be more accurately represented in a table where all the combinations of different links for each node were listed, compared to drawing it out. Redundant nodes were also more easily found by using this representation. Furthermore, the ónowflakeô geometrical representation was proposed to illustrate the connectivity of nodes in CHR6o3 as well as to aid the proving of some properties involving its Hamiltonicity. The results of this research are important in developing a routing algorithm for CHR6o3.
\end{abstract}

Keyword: Degree six chordal rings; Geometrical representation; Tree visualisation; Optimal diameter; Optimal average path length 